IJMMS 2003:2, 109-117

PII. S0161171203007646

http://ijmms.hindawi.com

(c) Hindawi Publishing Corp.

\title{
LOCAL COMPACTNESS IN APPROACH SPACES II
}

\section{R. LOWEN and C. VERBEECK}

Received 20 May 2001

\begin{abstract}
This paper studies the stability properties of the concepts of local compactness introduced by the authors in 1998. We show that all of these concepts are stable for contractive, expansive images and for products.
\end{abstract}

2000 Mathematics Subject Classification: 54A05, 54B10, 54D45.

1. Introduction. Having introduced notions of local compactness, basis local compactness and related measures, and having studied the basic relationship among these concepts in [3], in this paper we study stability properties. Keeping in mind the stability properties of local compactness in TOP, the category of topological spaces and continuous maps, we study how our notions behave under mappings and products. Especially, in the topological case, the maps which are required in order to preserve local compactness are continuous open surjections. This necessitated first finding out what is the right notion of open map in AP. This is duly done in this paper, and it turns out that the notion which we use has nice characterizations for a number of primitive approach structures. Of course, it also turns out to be the right concept in connection with local compactness.

2. Properties of local compactness in AP. In the construct of topological spaces, we have the following properties related to local compactness (see any good textbook on topology, e.g., [4]).

THEOREM 2.1. If $f$ is a surjective, continuous, open map of a topological space $(X, \tau)$ to a topological space $\left(Y, \tau^{\prime}\right)$ and $(X, \tau)$ is (basis) locally compact, then so is $\left(Y, \tau^{\prime}\right)$.

THEOREM 2.2. Let $\left(\left(X_{i}, \tau_{i}\right)\right)_{i \in I}$ be a family of topological spaces. Then $\prod_{i \in I}\left(X_{i}, \tau_{i}\right)$ is (basis) locally compact if and only if each $\left(X_{i}, \tau_{i}\right)$ is (basis) locally compact and all but finitely many $\left(X_{i}, \tau_{i}\right)$ are compact.

We would like to investigate whether the LC $n$ and BLC $n$ admit similar properties in AP. First, however, we will have to define a concept in AP similar to the concept of an open map in TOP.

DeFinITION 2.3. Let $(X, \mathscr{A})$ and $\left(Y, \mathscr{A}^{\prime}\right)$ be approach spaces, let $f: X \rightarrow Y$ be a map, and let $x \in X$. Then $f$ is expansive at $x$ if and only if for every 
$\varphi \in \mathscr{A}(x), f(\varphi)$ belongs to $\mathscr{A}^{\prime}(f(x))$, where $f(\varphi)(y):=\inf _{f(z)=y} \varphi(z)$. The map $f$ is called an expansion if and only if it is expansive at every $x \in X$. In that case, we also say that $f$ is expansive.

REMARK 2.4. Let $(X, \mathscr{A})$ and $\left(Y, \mathscr{A}^{\prime}\right)$ be approach spaces, $f: X \rightarrow Y$ a map, and $\Lambda$ an approach basis for $\mathscr{A}$ and $x \in X$. Then the following properties are equivalent:

(1) $f$ is expansive at $x$;

(2) for all $\varphi \in \mathscr{A}(x)$, there exists $\varphi^{\prime} \in \mathscr{A}^{\prime}(f(x)): f(\varphi) \leq \varphi^{\prime}$;

(3) for all $\varphi \in \mathscr{A}(x)$, for all $\varepsilon, N \in] 0, \infty\left[\right.$, there exists $\varphi^{\prime} \in \mathscr{A}^{\prime}(f(x)): f(\varphi) \wedge$ $N \leq \varphi^{\prime}+\varepsilon$

(4) for all $\varphi \in \Lambda(x): f(\varphi) \in \mathscr{A}^{\prime}(f(x))$;

(5) for all $\varphi \in \Lambda(x)$, there exists $\varphi^{\prime} \in \mathscr{A}^{\prime}(f(x)): f(\varphi) \leq \varphi^{\prime}$;

(6) for all $\varphi \in \Lambda(x)$, for all $\varepsilon, N \in] 0, \infty\left[\right.$, there exists $\varphi^{\prime} \in \mathscr{A}^{\prime}(f(x)): f(\varphi) \wedge$ $N \leq \varphi^{\prime}+\varepsilon$.

Proposition 2.5. Let $(X, \tau)$ and $\left(Y, \tau^{\prime}\right)$ be topological spaces. Then $f$ : $\left(X, \mathscr{A}_{\tau}\right) \rightarrow\left(Y, \mathscr{A}_{\tau^{\prime}}\right)$ is expansive if and only if $f:(X, \tau) \rightarrow\left(Y, \tau^{\prime}\right)$ is open.

Proof. The proof goes as follows:

$$
\begin{aligned}
f \text { is an expansion } & \Longleftrightarrow \forall x \in X, \forall V \in \mathscr{V}(x): f\left(\theta_{V}\right) \in \mathscr{A}^{\prime}(f(x)) \\
& \Longleftrightarrow \forall x \in X, \forall V \in \mathscr{V}(x): \theta_{f(V)} \in \mathscr{A}^{\prime}(f(x)) \\
& \Longleftrightarrow \forall x \in X, \forall V \in \mathscr{V}(x): f(V) \in \mathscr{V}^{\prime}(f(x)) \\
& \Longleftrightarrow f \text { is open. }
\end{aligned}
$$

In a similar fashion, we can prove the following result.

Proposition 2.6. Let $(X, \mathscr{A})$ and $\left(Y, A^{\prime}\right)$ be approach spaces. If $f:(X, \mathscr{A}) \rightarrow$ $\left(Y, A^{\prime}\right)$ is an expansion, then $f:\left(X, \tau_{\mathscr{A}}\right) \rightarrow\left(Y, \tau_{\mathscr{A}^{\prime}}\right)$ is open.

Proposition 2.7. Let $(X, \mathscr{A})$ and $\left(Y, A^{\prime}\right)$ be approach spaces and let $f: X \rightarrow$ $Y$ be a map. Consider the following properties:

(1) $f$ is an expansion;

(2) for all $B \subset Y$, for all $\varepsilon \in[0, \infty]: f^{-1}\left(B^{(\varepsilon)^{\prime}}\right) \subset\left(f^{-1}(B)\right)^{(\varepsilon)}$;

(3) for all $B \subset Y: \delta_{f^{-1}(B)} \leq \delta_{B}^{\prime} \circ f$;

(4) for every filter $G$ on $Y$ with $G \cap f(X) \neq \varnothing$, for all $G \in G$ : $\lambda\left(\operatorname{stack} f^{-1}(\mathscr{G})\right) \leq$ $\lambda^{\prime}(G) \circ f$. The following relation holds:

$$
(1) \Longleftrightarrow(2) \Longleftrightarrow(3) \Longrightarrow(4) \text {. }
$$

If $f: X \rightarrow Y$ is surjective, then

$$
(1) \Longleftrightarrow(2) \Longleftrightarrow(3) \Longleftrightarrow\left(4^{\prime}\right)
$$

with

$\left(4^{\prime}\right)$ for every filter $\mathscr{G}$ on $Y, \lambda\left(\operatorname{stack} f^{-1}(\mathscr{G})\right) \leq \lambda^{\prime}(\mathscr{G}) \circ f$. 
Proof. (1) $\Rightarrow(2)$. Let $B \subset Y$ and $\varepsilon \in[0, \infty]$,

$$
\begin{aligned}
f^{-1}\left(B^{(\varepsilon)^{\prime}}\right) & =\left\{x \in X \mid \sup _{\varphi^{\prime} \in \mathscr{A}^{\prime}(f(x))} \inf _{b \in B} \varphi^{\prime}(b) \leq \varepsilon\right\} \\
& \subset\left\{x \in X \mid \sup _{\varphi \in \mathscr{A}(x)} \inf _{b \in B} f(\varphi)(b) \leq \varepsilon\right\} \\
& =\left\{x \in X \mid \sup _{\varphi \in \mathscr{A}(x)} \inf _{z \in f^{-1}(B)} \varphi(z) \leq \varepsilon\right\} \\
& =\left(f^{-1}(B)\right)^{(\varepsilon)} .
\end{aligned}
$$

(2) $\Rightarrow(3)$. Let $B \subset Y, x \in X$, and $\varepsilon>0$. Suppose $\delta^{\prime}(f(x), B) \leq \varepsilon$, then $x \in$ $f^{-1}\left(B^{(\varepsilon)^{\prime}}\right) \subset\left(f^{-1}(B)\right)^{(\varepsilon)}$, so $\delta\left(x, f^{-1}(B)\right) \leq \varepsilon$. This implies (3).

(3) $\Rightarrow(1)$. Suppose there exist an $x_{0} \in X$, a $\varphi_{0} \in \mathscr{A}\left(x_{0}\right)$, and $\left.\varepsilon, N \in\right] 0, \infty[$ such that for every $\varphi^{\prime} \in \mathscr{A}^{\prime}\left(f\left(x_{0}\right)\right), f\left(\varphi_{0}\right) \wedge N \nless \varphi^{\prime}+\varepsilon$. For every $\varphi^{\prime} \in \mathscr{A}^{\prime}\left(f\left(x_{0}\right)\right)$, define

$$
B\left(\varphi^{\prime}\right):=\left\{y \in Y \mid f\left(\varphi_{0}\right)(y) \wedge N>\varphi^{\prime}(y)+\varepsilon\right\}
$$

Notice that for every $\varphi^{\prime}, \xi^{\prime} \in \mathscr{A}^{\prime}\left(f\left(x_{0}\right)\right), B\left(\varphi^{\prime} \vee \xi^{\prime}\right)=B\left(\varphi^{\prime}\right) \cap B\left(\xi^{\prime}\right)$ and $B\left(\varphi^{\prime}\right) \neq$ $\varnothing$. Then

$$
\begin{aligned}
\sup _{\varphi^{\prime} \in \mathscr{A}^{\prime}\left(f\left(x_{0}\right)\right)} \delta^{\prime} & \left(f\left(x_{0}\right), B\left(\varphi^{\prime}\right)\right) \wedge N \\
& =\sup _{\varphi^{\prime} \in \mathscr{A}^{\prime}\left(f\left(x_{0}\right)\right)} \sup _{\theta \in \mathscr{A}^{\prime}\left(f\left(x_{0}\right)\right)} \inf _{y \in B\left(\varphi^{\prime}\right)} \theta(y) \wedge N \\
& \leq \sup _{\varphi^{\prime} \in \mathscr{A}^{\prime}\left(f\left(x_{0}\right)\right)} \sup _{\theta \in \mathscr{A}^{\prime}\left(f\left(x_{0}\right)\right)} \inf _{y \in B\left(\varphi^{\prime} \vee \theta\right)}\left(\varphi^{\prime} \vee \theta\right)(y) \wedge N \\
& =\sup _{\varphi^{\prime} \in \mathscr{A}^{\prime}\left(f\left(x_{0}\right)\right)} \inf _{y \in B\left(\varphi^{\prime}\right)} \varphi^{\prime}(y) \wedge N \\
& \leq \sup _{\varphi^{\prime} \in \mathscr{A}^{\prime}\left(f\left(x_{0}\right)\right)} \inf _{y \in B\left(\varphi^{\prime}\right)}\left(f\left(\varphi_{0}\right)(y) \wedge N\right)-\varepsilon \\
& \leq \sup _{\varphi^{\prime} \in \mathscr{A}^{\prime}\left(f\left(x_{0}\right)\right)} \sup _{\xi \in \mathscr{A}\left(x_{0}\right)} \inf _{x \in f^{-1}\left(B\left(\varphi^{\prime}\right)\right)}(\xi(x) \wedge N)-\varepsilon \\
& =\sup _{\varphi^{\prime} \in \mathscr{A}^{\prime}\left(f\left(x_{0}\right)\right)}\left(\delta\left(x_{0}, f^{-1}\left(B\left(\varphi^{\prime}\right)\right)\right) \wedge N\right)-\varepsilon,
\end{aligned}
$$

so

$$
\sup _{\varphi^{\prime} \in \mathscr{A}^{\prime}\left(f\left(x_{0}\right)\right)} \delta^{\prime}\left(f\left(x_{0}\right), B\left(\varphi^{\prime}\right)\right)<\sup _{\varphi^{\prime} \in \mathscr{A}^{\prime}\left(f\left(x_{0}\right)\right)} \delta\left(x_{0}, f^{-1}\left(B\left(\varphi^{\prime}\right)\right)\right),
$$

which contradicts (3).

(3) $\Rightarrow(4)$. Let $\mathscr{G}$ be a filter on $Y$ such that $G \cap f(X) \neq \varnothing$ for all $G \in \mathscr{G}$,

$$
\lambda\left(\operatorname{stack} f^{-1}(\mathscr{G})\right)=\sup _{\mathscr{U} \in U\left(\operatorname{stack} f^{-1}(\mathscr{G})\right)} \sup _{U \in \mathscr{U}} \delta_{U}
$$


Now $\mathcal{U}$ is an ultrafilter containing stack $f^{-1}(\mathscr{G})$ if and only if $\mathscr{V}:=\operatorname{stack} f(\mathcal{U})$ is an ultrafilter containing $\mathscr{G}$. Hence,

$$
\begin{aligned}
\lambda\left(\operatorname{stack} f^{-1}(\mathscr{G})\right) & =\sup _{\mathscr{V} \in U(\mathscr{G})} \sup _{U \in \operatorname{stack} f^{-1}(\mathscr{V})} \delta_{U} \\
& =\sup _{\mathscr{V} \in U(\mathscr{G})} \sup _{V \in \mathscr{V}} \sup _{U \supset f^{-1}(V)} \delta_{U} .
\end{aligned}
$$

Suppose that $\mathscr{V}$ is an ultrafilter containing $\mathscr{G}, V \in \mathscr{V}$, and $U \supset f^{-1}(V)$. Then for every $x \in X, \delta(x, U) \leq \delta\left(x, f^{-1}(V)\right) \leq \delta^{\prime}(f(x), V)$, whence

$$
\begin{aligned}
\lambda\left(\operatorname{stack} f^{-1}(\mathscr{G})\right)(x) & =\sup _{\mathscr{V} \in U(\mathscr{G})} \sup _{V \in \mathscr{V}} \sup _{U \supset f^{-1}(V)} \delta_{U} \\
& \leq \sup _{\mathscr{V} \in U(\mathscr{\varphi})} \sup _{V \in \mathscr{V}} \delta^{\prime}(f(x), V)=\lambda^{\prime}(\mathscr{\varphi})(f(x)) .
\end{aligned}
$$

$\left(4^{\prime}\right) \Leftrightarrow(3)$. If $f$ is surjective, then $(4) \Leftrightarrow\left(4^{\prime}\right)$, so we are to prove (4) $\Rightarrow(3)$. If we take a subset $B$ of $Y$, then $\delta_{B}^{\prime} \circ f=\inf _{\mathscr{V} \in U(B)} \lambda^{\prime}(\mathscr{V}) \circ f$. Now suppose that $\mathscr{V}$ is an ultrafilter containing $B$, then stack $f^{-1}(\mathscr{V})$ is an ultrafilter containing stack $f^{-1}(B)$. We infer

$$
\begin{aligned}
\delta_{B}^{\prime} \circ f & \geq \inf _{\mathscr{V} \in U(B)} \lambda\left(\operatorname{stack} f^{-1}(\mathscr{V})\right) \\
& \geq \operatorname{stack}^{-1} \inf _{(\mathscr{V}) \in U\left(f^{-1}(B)\right)} \lambda\left(\operatorname{stack} f^{-1}(\mathscr{V})\right) \\
& \geq \inf _{\mathscr{U} \in U\left(f^{-1}(B)\right)} \lambda \mathscr{U}=\delta_{f^{-1}(B)} .
\end{aligned}
$$

Proposition 2.8. Let $\left(\left(X_{i}, \mathscr{A}_{i}\right)\right)_{i \in I}$ be a family of approach spaces and let $(X, A)$ be their product approach space. Then for every $k \in I$, the projection $\operatorname{pr}_{k}:(X, \mathscr{A}) \rightarrow\left(X_{k}, \mathscr{A}_{k}\right)$ is an expansion.

Proof. Let $x=\left(x_{i}\right)_{i \in I}$ be an element of $X$. Then an approach basis for $\mathscr{A}(x)$ is given by (see $[1,2])$

$$
\Lambda(x)=\left\{\sup _{j \in J} \varphi_{j} \circ \operatorname{pr}_{j} \mid J \in 2^{(I)}, \forall j \in J: \varphi_{j} \in \mathscr{A}_{j}\left(x_{j}\right)\right\} .
$$

Take $k \in I$, a finite subset $J$ of $I$, and a collection $\left(\varphi_{j}\right)_{j \in J}$ such that $\varphi_{j}$ belongs to $\mathscr{A}_{j}\left(x_{j}\right)$ for every $j \in J$. We need to prove that

$$
\operatorname{pr}_{k}\left(\sup _{j \in J} \varphi_{j} \circ \operatorname{pr}_{j}\right) \in \mathscr{A}_{k}\left(x_{k}\right)
$$

Let $y \in X_{k}$. Notice that $\operatorname{pr}_{k}\left(\sup _{j \in J} \varphi_{j} \circ \operatorname{pr}_{j}\right)(y)=\inf _{z_{k}=y} \sup _{j \in J} \varphi_{j}\left(z_{j}\right)$. 
First case: $k \in J$. If we define $z^{0}:=\left(z_{i}^{0}\right)_{i \in I}$ with $z_{j}^{0}:=x_{j}$ for every $j \in J \backslash\{k\}$, $z_{k}^{0}:=y$, and $z_{i}^{0}$ a fixed but arbitrary element of $X_{i}$ for every $i \in I \backslash J$, we get that

$$
\begin{aligned}
\inf _{z_{k}=y} \sup _{j \in J} \varphi_{j}\left(z_{j}\right) & =\inf _{z_{k}=y}\left(\varphi_{k}(y) \vee \sup _{j \in J \backslash\{k\}} \varphi_{j}\left(z_{j}\right)\right) \\
& =\varphi_{k}(y) \vee \inf _{z_{k}=y} \sup _{j \in J \backslash\{k\}} \varphi_{j}\left(z_{j}\right) \\
& \leq \varphi_{k}(y) \vee \sup _{j \in J \backslash\{k\}} \varphi_{j}\left(z_{j}^{0}\right) .
\end{aligned}
$$

Hence, $\operatorname{pr}_{k}\left(\sup _{j \in J} \varphi_{j} \circ \operatorname{pr}_{j}\right)(y) \leq \varphi_{k}(y) \vee 0=\varphi_{k}(y)$, which entails $\operatorname{pr}_{k}\left(\sup _{j \in J} \varphi_{j} \circ \operatorname{pr}_{j}\right) \in \mathscr{A}_{k}\left(x_{k}\right)$.

Second case: $k \notin J$. Define $z_{j}^{0}:=x_{j}$ for every $j \in J, z_{k}^{0}:=y$, and $z_{i}^{0}$ a fixed but arbitrary element of $X_{i}$ for every $i \in I \backslash(J \cup\{k\})$. Then

$$
\operatorname{pr}_{k}\left(\sup _{j \in J} \varphi_{j} \circ \mathrm{pr}_{j}\right)(y)=\inf _{z_{k}=y} \sup _{j \in J} \varphi_{j}\left(z_{j}\right) \leq \sup _{j \in J} \varphi_{j}\left(z_{j}^{0}\right)=0,
$$

so $\operatorname{pr}_{k}\left(\sup _{j \in J} \varphi_{j} \circ \operatorname{pr}_{j}\right)=0 \in \mathscr{A}_{k}\left(x_{k}\right)$.

We will now formulate and prove properties of local compactness in AP similar to Theorems 2.1 and 2.2 in TOP.

THEOREM 2.9. For every $n \in\{1,2,3,4,5\}$, if $f:(X, \mathscr{A}) \rightarrow\left(Y, A^{\prime}\right)$ is a surjective, expansive contraction and $(X, A)$ is $(B) L C n$, then $\left(Y, A^{\prime}\right)$ is $(B) L C n$ as well.

Proof. We will only prove the case of BLC5, as the other cases follow similar lines of thought. Let $y \in Y$, then there exists an $x \in X$ such that $y=f(x)$. Let $\varepsilon>0$. We first show that $\mathscr{V}_{\varepsilon}^{\prime}(y)=\operatorname{stack} f\left(\mathscr{V}_{\varepsilon}(x)\right)$, suppose $F$ belongs to $\mathscr{V}_{\varepsilon}(x)$. Then there exist some $\varphi \in \mathscr{A}(x)$ such that $F \supset\{\varphi<\varepsilon\}$, so since $f(\varphi)$ belongs to $\mathscr{A}^{\prime}(y), f(\{\varphi<\varepsilon\})=\{f(\varphi)<\varepsilon\}$ belongs to $\mathscr{V}_{\varepsilon}^{\prime}(y)$. Hence $f(F)$ belongs to $\mathscr{V}_{\varepsilon}^{\prime}(y)$. Next, suppose that $G \in \mathscr{V}_{\varepsilon}^{\prime}(y)$, then there exists some $\varphi^{\prime} \in \mathscr{A}^{\prime}(y)$ such that $G \supset\left\{\varphi^{\prime}<\varepsilon\right\}$. Since $\varphi^{\prime} \circ f$ belongs to $\mathscr{A}(x),\left\{\varphi^{\prime} \circ f<\varepsilon\right\} \in \mathscr{V}_{\varepsilon}(x)$, whence $f\left(\left\{\varphi^{\prime} \circ f<\varepsilon\right\}\right)=\left\{\varphi^{\prime}<\varepsilon\right\}$ belongs to $f\left(\mathscr{V}_{\varepsilon}(x)\right)$. This implies $G \in$ stack $f\left(\mathscr{V}_{\varepsilon}(x)\right)$.

This result being shown, take $G \in \mathscr{V}_{\varepsilon}(y)$ and $\varepsilon^{\prime}<\varepsilon$. Then

$$
\begin{aligned}
\inf _{\substack{H \in \mathscr{V}^{\prime} \varepsilon^{\prime}(y) \\
H \subset G}} \mu_{c}(H) \leq \inf _{\substack{K \in \mathcal{V}_{\varepsilon^{\prime}}(x) \\
K \subset f^{-1}(G)}} \mu_{c}(f(K)) \leq \inf _{\substack{K \in \mathscr{V}_{\varepsilon^{\prime}}(x) \\
K \subset f^{-1}(G)}} \mu_{c}(K) \\
\leq \inf _{z \in X} \lambda \mathcal{V}_{\varepsilon^{\prime}}(x)(z) \leq \inf _{w \in Y} \lambda^{\prime} \mathscr{V}_{\varepsilon^{\prime}}^{\prime}(y)(w) .
\end{aligned}
$$


For the notions (B)LC1 and (B)LC2, we are able to prove a theorem completely similar to Theorem 2.2. This is not possible, however, for the other notions of (basis) local compactness in AP. We do have a somewhat weaker result for these notions.

THEOREM 2.10. For every $n \in\{1,2\}$, let $\left(\left(X_{i}, A_{i}\right)\right)_{i \in I}$ be a family of approach spaces. Then $\prod_{i \in I}\left(X_{i}, \mathscr{A}_{i}\right)$ is (B)LCn if and only if each $\left(X_{i}, \mathscr{A}_{i}\right)$ is $(B) L C n$ and all but finitely many $\left(X_{i}, \tau_{s i}\right)$ are compact.

Proof. For (B)LC1, this theorem is a straightforward consequence of Theorem 2.2 and the fact that the topological coreflection of a product in AP is the product in TOP of the topological coreflections of the factor spaces. For (B)LC2, the proof is easy and almost identical to the proof of Theorem 2.2.

As we will see in the following theorems, the product theorem for (basis) local compactness in TOP breaks down in two parts for the notions (B)LC3, (B)LC4, and (B)LC5.

THeOREM 2.11. For every $n \in\{3,4,5\}$, let $\left(\left(X_{i}, \mathscr{A}_{i}\right)\right)_{i \in I}$ be a family of approach spaces. If $(X, \mathscr{A}):=\prod_{i \in I}\left(X_{i}, \mathscr{A}_{i}\right)$ is $(B) L C n$, then $\left(X_{i}, \mathscr{A}_{i}\right)$ is $(B) L C n$ for every $i \in I$ and there exists some countable $J \subset I$ such that for every $i \in I \backslash J$ : $\mu_{c}\left(X_{i}\right)=0$.

Proof. Suppose $(X, \mathscr{A})$ is $(\mathrm{B}) \mathrm{LC} n$. It follows immediately from Theorem 2.9 and Proposition 2.8 that $\left(X_{i}, \mathscr{A}_{i}\right)$ is (B)LC $n$ for every $i \in I$.

For the remaining part, suppose that $(X, \mathscr{A})$ is LC3. Let $x \in X$, then for every nonzero natural number $n$, there exists some neighborhood $V_{n}$ of $x$ in the topological coreflection such that $\mu_{c}\left(V_{n}\right) \leq n^{-1}$. Hence there exists some finite $J_{n} \subset I$ such that for every $i \in I \backslash J_{n}: \operatorname{pr}_{i}\left(V_{n}\right)=X_{i}$. Define $J:=\bigcup_{n} J_{n}$ and let $i \in I \backslash J$, then for every $n$,

$$
\mu_{c}\left(X_{i}\right)=\mu_{c}\left(\operatorname{pr}_{i}\left(V_{n}\right)\right) \leq \mu_{c}\left(V_{n}\right) \leq n^{-1},
$$

hence $\mu_{c}\left(X_{i}\right)=0$.

If $(X, \mathscr{A})$ is $\mathrm{BLC} 3,(\mathrm{~B}) \mathrm{LC} 4$, or $(\mathrm{B}) \mathrm{LC} 5$, it is also LC3 and we get the same result.

THEOREM 2.12. For every $n \in\{3,4,5\}$, let $\left(\left(X_{i}, A_{i}\right)\right)_{i \in I}$ be a family of approach spaces. If every $\left(X_{i}, \mathscr{A}_{i}\right)$ is $(B) L C n$ and if there exists some finite $J \subset I$ such that for all $i \in I \backslash J: \mu_{c}\left(X_{i}\right)=0$, then $(X, \mathscr{A}):=\prod_{i \in I}\left(X_{i}, \mathscr{A}_{i}\right)$ is (B)LCn.

Proof. We will prove this property for BLC5, as all other cases are similar and, moreover, simpler than this one.

Suppose every $\left(X_{i}, \mathscr{A}_{i}\right)$ is BLC5 and there exists some finite subset $J \subset I$ such that for all $i \in I \backslash J: \mu_{c}\left(X_{i}\right)=0$. Let $x \in X, \varepsilon>0$, and $F \in \mathscr{V}_{\varepsilon}(x)$. Then there 
exists some $\varphi \in \mathscr{A}(x)$ such that $F \supset\{\varphi<\varepsilon\}$. Take $\varepsilon^{\prime}<\varepsilon$ and $N>\varepsilon$. Then there exists some finite subset $K_{\varepsilon^{\prime}}^{N} \subset I$ such that for every $k \in K_{\varepsilon^{\prime}}^{N}$, there exists some $\varphi_{k, \varepsilon^{\prime}}^{N} \in \mathscr{A}_{k}\left(x_{k}\right)$ such that

$$
\varphi \wedge N \leq \sup _{k \in K_{\varepsilon^{\prime}}^{N}} \varphi_{k, \varepsilon^{\prime}}^{N} \circ \operatorname{pr}_{k}+\left(\varepsilon-\varepsilon^{\prime}\right)
$$

Now

$$
F \supset\left(\sup _{k \in K_{\varepsilon^{\prime}}^{N}} \varphi_{k, \varepsilon^{\prime}}^{N} \circ \operatorname{pr}_{k}+\left(\varepsilon-\varepsilon^{\prime}\right)<\varepsilon\right)=\left(\sup _{k \in K_{\varepsilon^{\prime}}^{N}} \varphi_{k, \varepsilon^{\prime}}^{N} \circ \operatorname{pr}_{k}<\varepsilon^{\prime}\right) .
$$

We will adopt a fixed value of $N$ and drop the superscript $N$ in $K_{\varepsilon^{\prime}}^{N}$ and $\varphi_{k, \varepsilon^{\prime}}^{N}$. Define

$$
F_{k, \varepsilon^{\prime}}:=\left\{\varphi_{k, \varepsilon^{\prime}}<\varepsilon^{\prime}\right\} \in \mathscr{V}_{\varepsilon^{\prime}}\left(x_{k}\right), \quad \forall k \in k_{\varepsilon^{\prime}}
$$

Let $\varepsilon^{\prime \prime}<\varepsilon^{\prime}$ and $\delta>0$, then for every element $k$ of $K_{\varepsilon^{\prime}}$, there exists some element $G_{k, \varepsilon^{\prime}, \varepsilon^{\prime \prime}, \delta}$ of $\mathscr{V}_{\varepsilon^{\prime \prime}}\left(x_{k}\right)$ such that $G_{k, \varepsilon^{\prime}, \varepsilon^{\prime \prime}, \delta} \subset F_{k, \varepsilon^{\prime}}$ and also such that $\mu_{c}\left(G_{k, \varepsilon^{\prime}, \varepsilon^{\prime \prime}, \delta}\right) \leq \inf _{y \in X_{k}} \lambda_{k} \mathscr{V}_{\varepsilon^{\prime \prime}}\left(x_{k}\right)(y)+\delta$, for $\left(X_{k}, A_{k}\right)$ is BLC5. This means that for every $k \in K_{\varepsilon^{\prime}}$, there exists, some element $\psi_{k, \varepsilon^{\prime}, \varepsilon^{\prime \prime}, \delta} \in \mathscr{A}_{k}\left(x_{k}\right)$ such that $G_{k, \varepsilon^{\prime}, \varepsilon^{\prime \prime}, \delta} \supset\left\{\psi_{k, \varepsilon^{\prime}, \varepsilon^{\prime \prime}, \delta}<\varepsilon^{\prime \prime}\right\}$. Now for every $j \in J$, there is an $F_{j, \varepsilon^{\prime}, \varepsilon^{\prime \prime}, \delta}^{\prime} \in \mathscr{V}_{\varepsilon^{\prime \prime}}\left(x_{j}\right)$ such that $\mu_{c}\left(F_{j, \varepsilon^{\prime}, \varepsilon^{\prime \prime}, \delta}^{\prime}\right) \leq \inf _{y \in X_{j}} \lambda_{j} \mathscr{V}_{\varepsilon^{\prime \prime}}\left(x_{j}\right)(y)+\delta$, as $\left(X_{j}, \mathscr{A}_{j}\right)$ is LC5. Consequently, for every $j \in J$, there exists some $\psi_{j, \varepsilon^{\prime}, \varepsilon^{\prime \prime}, \delta}^{\prime} \in \mathscr{A}_{j}\left(x_{j}\right)$ such that $F_{j, \varepsilon^{\prime}, \varepsilon^{\prime \prime}, \delta}^{\prime} \supset\left\{\psi_{j, \varepsilon^{\prime}, \varepsilon^{\prime \prime}, \delta}^{\prime}<\varepsilon^{\prime \prime}\right\}$. If we define

$$
H_{\varepsilon^{\prime}, \varepsilon^{\prime \prime}, \delta}:=\prod_{k \in K_{\varepsilon^{\prime}}} G_{k, \varepsilon^{\prime}, \varepsilon^{\prime \prime}, \delta} \times \prod_{j \in J \backslash K_{\varepsilon^{\prime}}} F_{j, \varepsilon^{\prime}, \varepsilon^{\prime \prime}, \delta}^{\prime} \times \prod_{i \in I \backslash\left(J \cup K_{\varepsilon^{\prime}}\right)} X_{i},
$$

then $H_{\varepsilon^{\prime}, \varepsilon^{\prime \prime}, \delta} \subset F$. Moreover, $H_{\varepsilon^{\prime}, \varepsilon^{\prime \prime}, \delta}$ belongs to $\mathscr{V}_{\varepsilon^{\prime \prime}}(x)$.

Suppose that

$$
y \in\left\{\sup _{k \in K_{\varepsilon^{\prime}}} \psi_{k, \varepsilon^{\prime}, \varepsilon^{\prime \prime}, \delta} \circ \mathrm{pr}_{k} \vee \sup _{j \in J \backslash K_{\varepsilon^{\prime}}} \psi_{j, \varepsilon^{\prime}, \varepsilon^{\prime \prime}, \delta}^{\prime} \circ \mathrm{pr}_{j}<\varepsilon^{\prime \prime}\right\} .
$$

Then for every $k \in K_{\varepsilon^{\prime}}, \psi_{k, \varepsilon^{\prime}, \varepsilon^{\prime \prime}, \delta}\left(y_{k}\right)<\varepsilon^{\prime \prime}$ and for every $j \in J \backslash K_{\varepsilon^{\prime}}, \psi_{j, \varepsilon^{\prime}, \varepsilon^{\prime \prime}, \delta}^{\prime}\left(y_{j}\right)$ $<\varepsilon^{\prime \prime}$, so for every $k \in K_{\varepsilon^{\prime}}: y_{k} \in G_{k, \varepsilon^{\prime}, \varepsilon^{\prime \prime}, \delta}$ and for every $j \in J \backslash K_{\varepsilon^{\prime}}: y_{j} \in F_{j, \varepsilon^{\prime}, \varepsilon^{\prime \prime}, \delta}^{\prime}$. 
This shows $y \in H_{\varepsilon^{\prime}, \varepsilon^{\prime \prime}, \delta}$, whence $H_{\varepsilon^{\prime}, \varepsilon^{\prime \prime}, \delta} \in \mathscr{V}_{\varepsilon^{\prime \prime}}(x)$,

$$
\begin{aligned}
& \mu_{c}\left(H_{\varepsilon^{\prime}, \varepsilon^{\prime \prime}, \delta}\right)=\sup _{k \in K_{\varepsilon^{\prime}}} \mu_{c}\left(G_{k, \varepsilon^{\prime}, \varepsilon^{\prime \prime}, \delta}\right) \vee \sup _{j \in J \backslash K_{\varepsilon^{\prime}}} \mu_{c}\left(F_{j, \varepsilon^{\prime}, \varepsilon^{\prime \prime}, \delta}^{\prime}\right) \vee \sup _{i \in I \backslash\left(J \cup K_{\varepsilon^{\prime}}\right)} \mu_{c}\left(X_{i}\right) \\
& \leq\left(\sup _{k \in K_{\varepsilon^{\prime}}} \inf _{y \in X_{k}} \lambda_{k} \mathscr{V}_{\varepsilon^{\prime \prime}}\left(x_{k}\right)(y) \vee \sup _{j \in J \backslash K_{\varepsilon^{\prime}}} \inf _{y \in X_{j}} \lambda_{j} \mathscr{V}_{\varepsilon^{\prime \prime}}\left(x_{j}\right)(y)\right)+\delta \\
& \leq \sup _{l \in J \cup K_{\varepsilon^{\prime}}} \inf _{y \in X_{l}} \lambda_{l} \mathscr{V}_{\varepsilon^{\prime \prime}}\left(x_{l}\right)(y)+\delta
\end{aligned}
$$

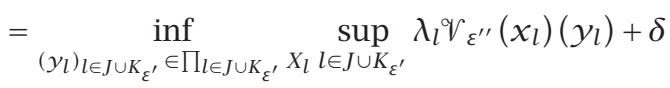

$$
\begin{aligned}
& =\inf _{y \in X} \sup _{l \in J \cup K_{\varepsilon^{\prime}}} \lambda_{l} \mathscr{V}_{\varepsilon^{\prime \prime}}\left(x_{l}\right)\left(y_{l}\right)+\delta \\
& \leq \inf _{y \in X} \sup _{i \in I} \lambda_{i} \mathscr{V}_{\varepsilon^{\prime \prime}}\left(x_{i}\right)\left(y_{i}\right)+\delta \\
& =\inf _{y \in X} \lambda \mathscr{V}_{\varepsilon^{\prime \prime}}(x)(y)+\delta
\end{aligned}
$$

So for every $\varepsilon^{\prime}<\varepsilon$ and for every $\varepsilon^{\prime \prime}<\varepsilon^{\prime}$,

$$
\inf _{\substack{H \in \mathscr{V}_{\varepsilon^{\prime \prime}}(x) \\ H \subset F}} \mu_{c}(H) \leq \inf _{y \in X} \lambda \mathscr{V}_{\varepsilon^{\prime \prime}}(x)(y),
$$

which implies that for every $\varepsilon^{\prime}<\varepsilon$,

$$
\inf _{\substack{H \in \mathcal{V}_{\varepsilon^{\prime}}(x) \\ H \subset F}} \mu_{c}(H) \leq \inf _{y \in X} \lambda \mathscr{V}_{\varepsilon^{\prime}}(x)(y)
$$

The proofs of the following theorems are an easy generalization of the proofs of the corresponding theorems for (basis) local compactness.

THEOREM 2.13. For every $n \in\{3,4,5\}$. If $f:(X, \mathscr{A}) \rightarrow\left(Y, \mathscr{A}^{\prime}\right)$ is a surjective, expansive contraction, then $\mu_{(B) L C n}(Y) \leq \mu_{(B) L C n}(X)$.

THEOREM 2.14. For every $n \in\{3,4,5\}$, let $\left(\left(X_{i}, \mathscr{A}_{i}\right)\right)_{i \in I}$ be a family of approach spaces and $(X, \mathscr{A}):=\prod_{i \in I}\left(X_{i}, \mathscr{A}_{i}\right)$. Then

$$
\mu_{(B) L C n}(X)=\sup _{i \in I} \mu_{(B) L C n}\left(X_{i}\right) \vee \inf _{J \text { finite } \subset I} \sup _{i \in I \backslash J} \mu_{\mathcal{C}}\left(X_{i}\right) .
$$

\section{REFERENCES}

[1] R. Lowen, Approach spaces: a common supercategory of TOP and MET, Math. Nachr. 141 (1989), 183-226.

[2] _ _ Approach Spaces. The Missing Link in the Topology-Uniformity-Metric Triad, Oxford Mathematical Monographs, The Clarendon Press, Oxford University Press, New York, 1997. 
[3] R. Lowen and C. Verbeeck, Local compactness in approach spaces. I, Int. J. Math. Math. Sci. 21 (1998), no. 3, 429-438.

[4] S. Willard, General Topology, Addison-Wesley, Massachusetts, 1970.

R. Lowen: Department of Mathematics, University of Antwerp, RUCA, Groenenborgerlaan 171, B-2020 Antwerp, Belgium

E-mail address: rlow@ruca.ua.ac. be

C. Verbeeck: Department of Mathematics and Computer Science, University of Antwerp, UIA, Universiteitsplein 1, B-2610 Antwerpen (Wilrijk), Belgium 


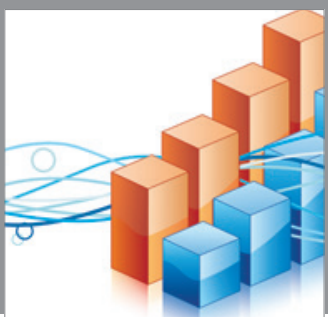

Advances in

Operations Research

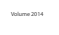

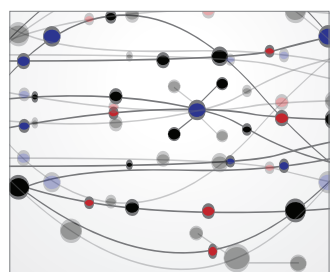

\section{The Scientific} World Journal
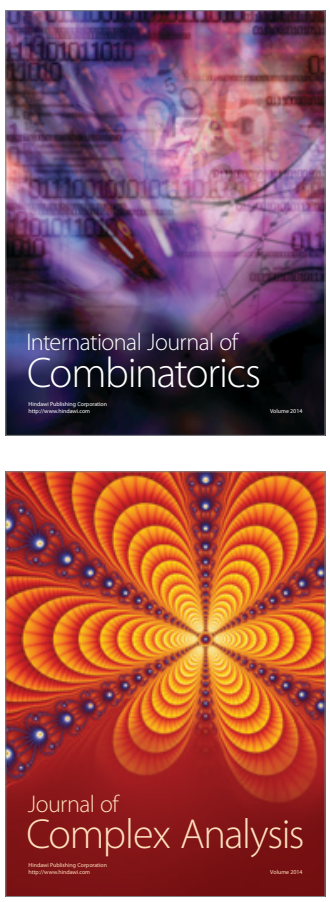

International Journal of

Mathematics and

Mathematical

Sciences
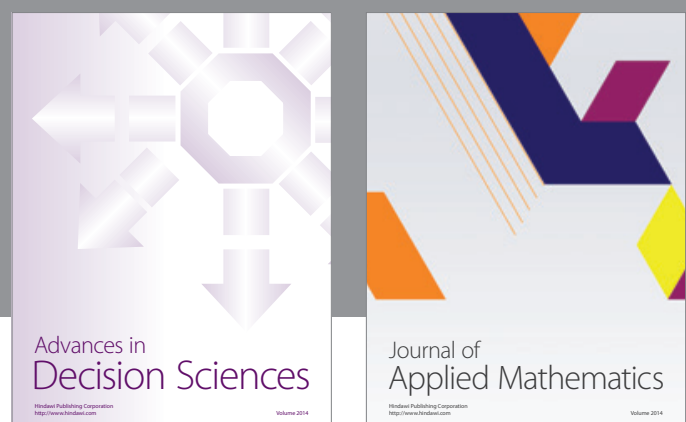

Journal of

Applied Mathematics
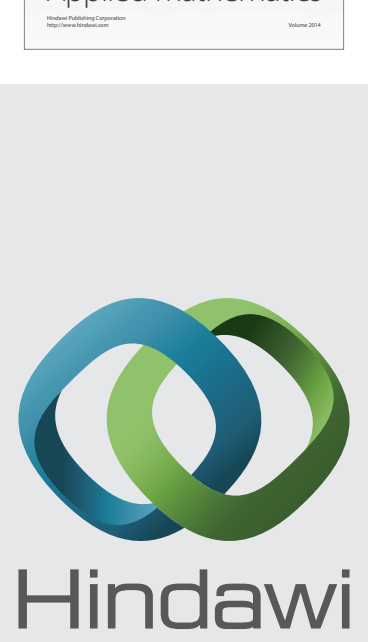

Submit your manuscripts at http://www.hindawi.com
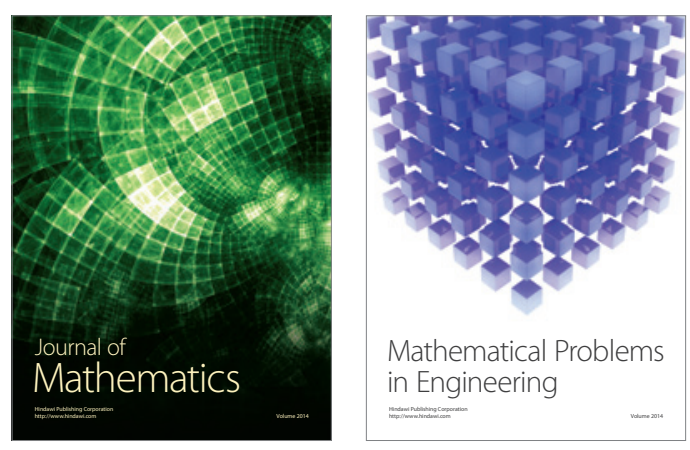

Mathematical Problems in Engineering
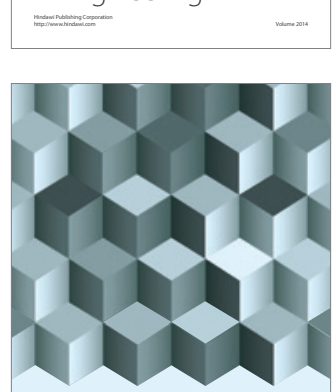

Journal of

Function Spaces
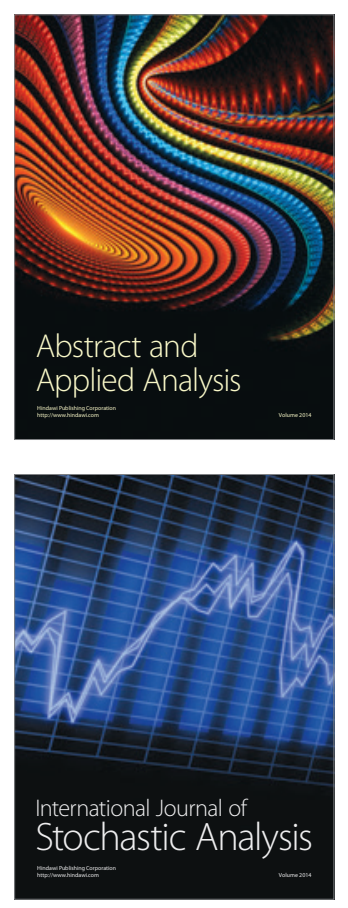

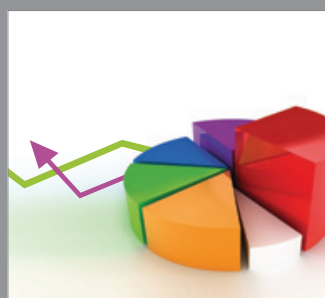

ournal of

Probability and Statistics

Promensencen
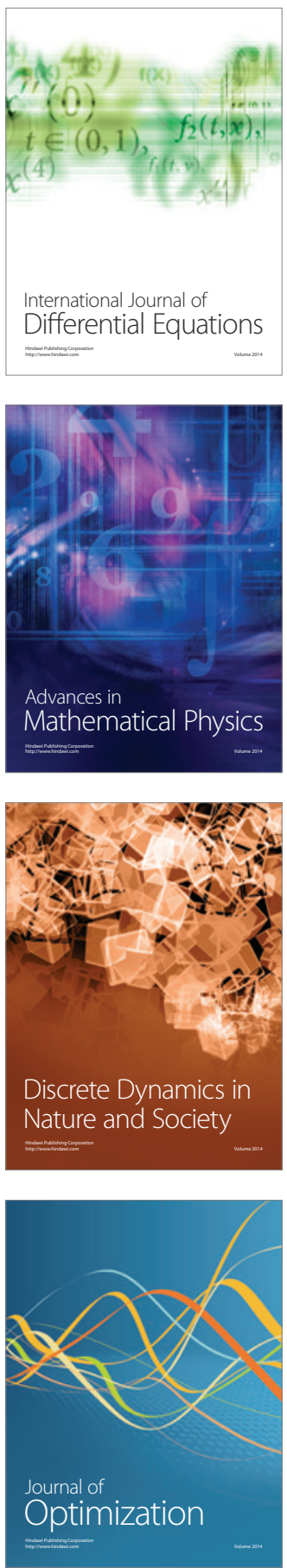